\title{
Metodologia Iterativa e Modelos Integradores para Desenvolvimento de Jogos Sérios de Treinamento e Avaliação de Desempenho Humano
}

\author{
Rafaela Vilela da Rocha (autora) ${ }^{1}$, Regina Borges de Araujo (orientadora) ${ }^{1}$ \\ ${ }^{1}$ Departamento de Computação - Universidade Federal de São Carlos (UFSCar) \\ Caixa Postal 676 - 13565-905 - São Carlos -SP - Brasil \\ \{rafaela_rocha, regina\}@dc.ufscar.br
}

\begin{abstract}
This paper describes an iterative methodology and integrative modes to develop serious games, integrating in a holistic and multidisciplinary approach the areas of simulation, game, learning, training and domain. The qualitative evaluation was conducted through interviews with five professionals from related areas and a feasibility study was done creating the serious game, which was used and evaluated by eight learners and two domain experts. In addition to the positive evaluation results, the serious games allowed evaluating an opportunity for improvement in the protocol.
\end{abstract}

Resumo. Este artigo descreve uma metodologia iterativa e modelos integradores para o desenvolvimento de jogos sérios, integrando de forma holística e multidisciplinar as áreas de simulação, jogo, aprendizagem, treinamento e domínio de aplicação. A avaliação qualitativa foi realizada por meio de entrevistas com cinco profissionais das áreas relacionadas e um estudo de viabilidade foi feito com a criação de um jogo sério, e seu uso e avaliação por oito aprendizes e dois especialistas no domínio. Além dos resultados positivos nas avaliações, a criação do jogo sério, com a aplicação da metodologia, permitiu avaliar uma oportunidade de melhoria no protocolo operacional utilizado.

\section{Introdução}

Um treinamento visa aprimorar as competências dos profissionais (conhecimento, habilidades e atitudes) e é essencial particularmente em atividades que envolvem risco à vida, ao patrimônio e ao meio ambiente. Devido a estes riscos e ao custos dos treinamentos práticos tradicionais, jogos sérios vêm sendo desenvolvidos, utilizados e integrados em diversos treinamentos. Entretanto, o desenvolvimento de jogos sérios é um processo complexo, com alto custo (de recursos humanos, materiais, financeiros, espaço e tempo). Rocha [2014], discute sete desafios nesta área relacionados às: características do produto final; inclusão de competências a serem treinadas; integração de diferentes profissionais no desenvolvimento; sistematização e padronização de artefatos e processos no desenvolvimento; reuso e extensão destes artefatos; avaliação e validação, tanto do aprendizado quanto do jogo sério. Para oferecer suporte para o desenvolvimento de jogos sérios, superando estes desafios, diferentes metodologias emergiram, além das existentes nas áreas inter-relacionadas. Entretanto, de forma geral, estas metodologias não descrevem o ciclo de vida completo, compreendendo os 
múltiplos requisitos dos Jogos Sérios para fins de Treinamento e Avaliação de desempenho humano (JSTAs). Muitas metodologias são focadas em áreas específicas e apenas enfatizam elementos de jogo, ou da simulação, ou da instrução/treinamento. Outras unificam alguns elementos destas áreas, mas sem abranger todos os requisitos necessários, criando uma lacuna entre elas.

Neste contexto, este artigo descreve uma nova metodologia, nomeada DevJSTA, que visa possibilitar o desenvolvimento de jogos sérios para fins de treinamento de competências operacionais baseadas em procedimentos-padrão e avaliação de desempenho humano. Na seção 2 é apresentado o estado da arte de metodologias de desenvolvimento. A seção 3 apresenta a metodologia de desenvolvimento DevJSTA e os modelos integradores. Na seção 4 são apresentados as avaliações e os resultados, seguidas das considerações finais, na seção 5 .

\section{Trabalhos Relacionados}

Uma revisão da literatura foi realizada para obter uma compreensão geral do estado da arte e as limitações das metodologias existentes. Foram pesquisados artigos nos principais motores de busca, em congressos e periódicos das áreas relacionadas; e livros e sites de referência que descrevem metodologias de desenvolvimento de jogos sérios e produtos relacionados; além de consultadas as referências citadas nesses trabalhos.

Elas podem ser agrupadas em metodologias de: (1) Design de jogos de entretenimento [Chandler, 2012; Godoy, 2010; Laubisch e Clua, 2010; Novak, 2010; Schuytema, 2008]; (2) Modelagem de simulações [Balci, 2012; Banks et al., 2001; IEEE, 2003 e 2010; Ford, 2004]; (3) Design de instruções e treinamentos [ABNT, 2001; Dick, Carey e Carey, 2004]; (4) Design de simulações e jogos educacionais [Akilli e Cagiltay, 2006; Adams et al, 2007; Aslan e Balci, 2015; Hays, 2005; Zin, Jaafar e Yue, 2009]; e (5) Desenvolvimento de jogos sérios [Becker e Parker, 2012; Freitas e Jarvis, 2006; Kirkley, Tomblin e Kirkley, 2005; Rodrigues, Machado e Valença, 2010; Van Der Zee, Holkenborgb e Robinson, 2012], conforme descrição e análise completa apresentada em Rocha [2014]. Suas limitações são descritas a seguir.

De forma geral, nas metodologias de desenvolvimento de jogos faltam detalhamentos/ documentações dos processos e dos artefatos produzidos; e faltam inclusões de requisitos de simulação, aprendizagem, treinamento e avaliação. Ao passo que, nas metodologias de modelagem e simulação: faltam detalhamentos dos requisitos instrucionais, de treinamento, de avaliação e do modelo de representação. Por outro lado, as especificações de design instrucional e de treinamento não abrangem requisitos de: jogos e simulações; e há diferenças significativas entre materiais educacionais e jogos sérios, por ex., linearidade, conteúdos estruturados e lógicos para auxiliar o instrutor, ênfase no processo em vez de foco no aprendizado e aprendizes. Já as metodologias de criação de simulações e jogos educacionais não oferecem apoio para avaliação e feedback do desempenho do aprendiz. Ao passo que, as metodologias de desenvolvimento de jogos sérios têm foco no design instrucional e do jogo, porém sem integração detalhada na metodologia, e não apoiam a avaliação do jogo sério.

\section{Metodologia DevJSTA e Modelos Integradores}

A DevJSTA abrange desde o planejamento e análise de jogos sérios até a avaliação das competências treinadas e do próprio jogo, integrando de forma holística e 
CBIE-LACLO 2015

Anais dos Workshops do IV Congresso Brasileiro de Informática na Educação (CBIE 2015)

multidisciplinar os requisitos de jogo, simulação, aprendizagem, treinamento $\mathrm{e}$ avaliação com o domínio de aplicação. Os conceitos e teorias que foram utilizados para integrar estas áreas são apresentados na Figura 1.

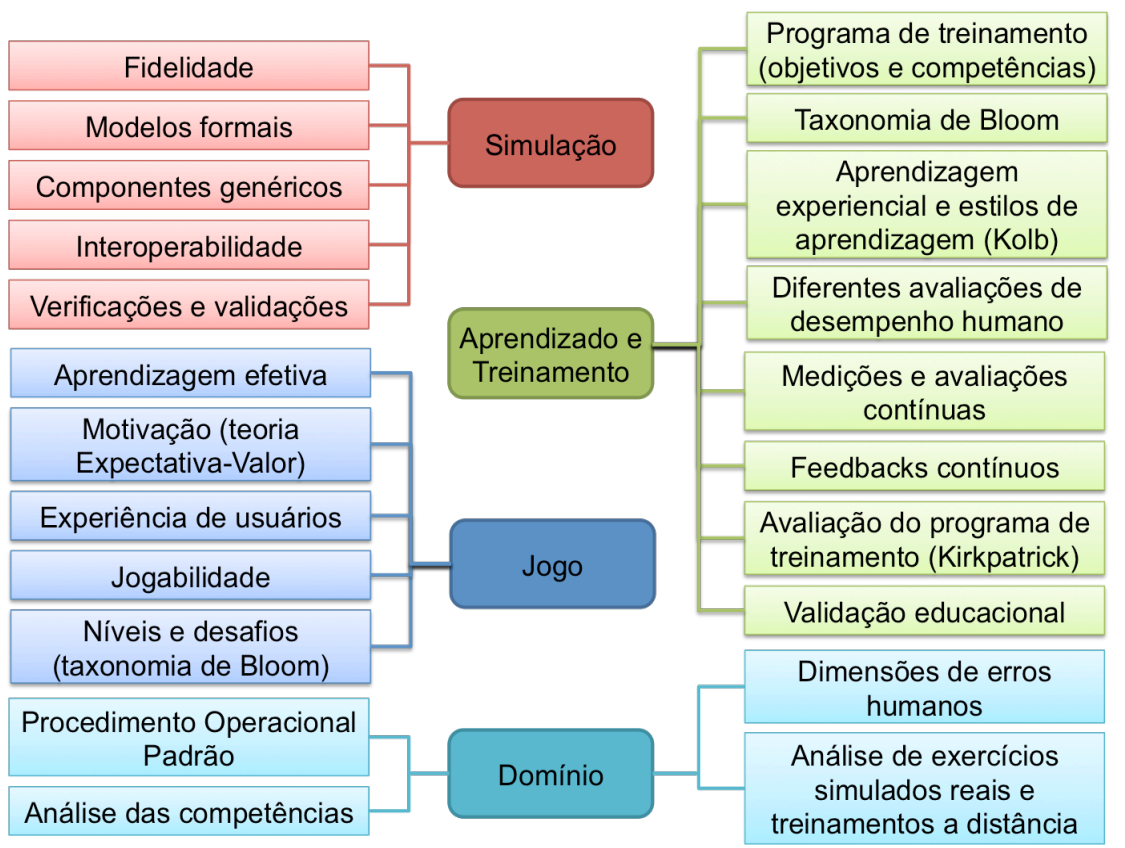

Figura 1 - Visão geral dos conceitos integrados por área.

\subsection{Processos da Metodologia DevJSTA}

A seguir são descritos os objetivos de cada processo da metodologia (Figura 2).

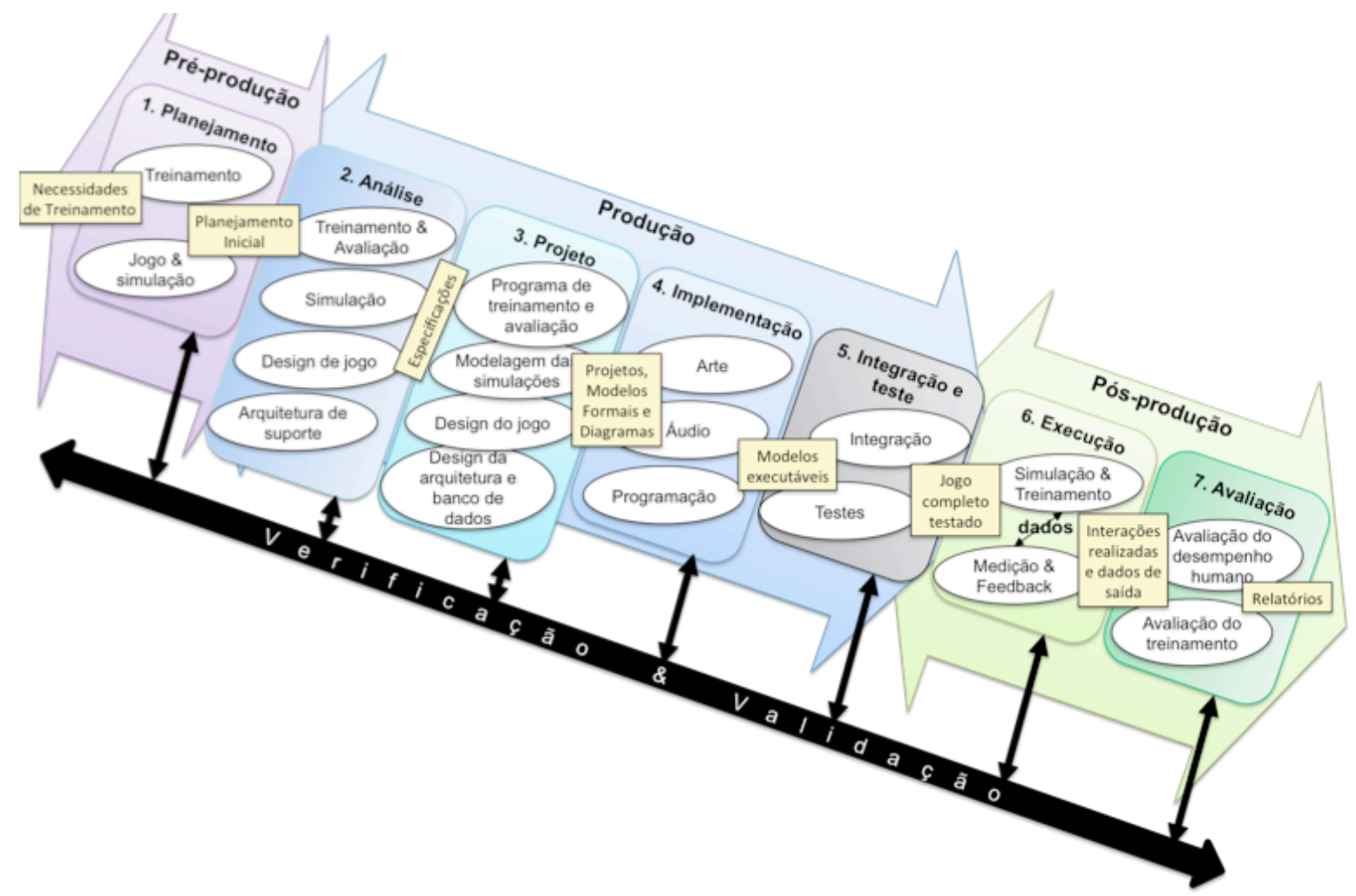

Figura 2. Metodologia de desenvolvimento de jogos sérios [adotado de Rocha, 2014]. 


\section{CBIE-LACLO 2015}

Anais dos Workshops do IV Congresso Brasileiro de Informática na Educação (CBIE 2015)

O processo (1) Planejamento (Pré-produção) visa a elaboração do plano inicial, a partir das necessidades de treinamento, contendo a situação do mundo real que será simulada, os procedimentos e as competências que serão treinadas. Este plano deve ser feito pelos responsáveis técnicos, que pode ser um profissional ou uma equipe que contém um ou mais pedagogo, professor, treinador e/ou especialista no domínio.

A etapa de Produção compreende os processos de 2 a 5. Na (2) Análise são especificados os requisitos para criar o jogo sério: incluindo dados do jogo, da simulação, e da arquitetura de suporte (definidos pelo analista de sistema), e do treinamento e avaliação (definidos pelo responsável técnico). Em (3) Projeto, os requisitos de jogo, simulação, arquitetura, treinamento e avaliação são transformados em projetos de jogo (responsabilidade do designer), simulação e arquitetura (responsabilidade do analista de sistema), e programa de treinamento (responsável técnico). Em (4) Implementação, os projetos do processo anterior são implementados pela equipe desenvolvedora, apoiados pelo responsável técnico. Esta equipe é formada por um ou mais programador e designer de arte e som, ou os respectivos responsáveis pelo desenvolvimento do código-fonte, da arte e do som (que podem incluir desde modeladores, animadores, produtores de som, até atores de voz e testadores). Em (5) Integração e Teste, os artefatos são integrados e testados até a conclusão do JS. Os profissionais da equipe desenvolvedora realizam suas funções para integrar e testar os recursos desenvolvidos no processo anterior.

Durante a Pós-produção, no processo (6) Execução, os aprendizes realizam o treinamento e, ao final, fazem uma autoavaliação (usando o questionário descrito em Rocha [2014]). O instrutor é responsável por acompanhar e auxiliar os aprendizes. Já no processo (7) Avaliação, o instrutor é responsável por gerar os relatórios de desempenho, realizar sua avaliação e fornecer o feedback aos aprendizes.

Ao final de cada processo, a atividade de verificação e validação deve ser conduzida pela equipe desenvolvedora, em conjunto com o responsável técnico. Caso seja necessário, o desenvolvimento pode voltar em algum processo anterior, em um processo iterativo e incremental.

Além da metodologia, também foram criados artefatos (por exemplo, formulários e critérios), modelos integradores (por exemplo, integrações de simulaçãoavaliação, jogo- simulação e programa de treinamento- avaliação), componentes e uma arquitetura de suporte que guiam e apoiam cada processo do ciclo de vida.

\subsection{Modelos Integradores}

Os Modelos Integradores (MIs) simulação-avaliação são um conjunto de modelos formais (DEVS - Especificação de evento discreto, DFA - Autômato determinístico finito, e FIS - Sistema de Inferência Fuzzy) e seus componentes, que processam dados e entradas para simular e avaliar os cenários de treinamento. Eles possibilitam a modelagem do comportamento do procedimento de treinamento e a inclusão dos pontos de avaliação do desempenho (principalmente a realização da sequência do procedimento é avaliada usando estes modelos formais). Os estados e transições destes modelos são pontos de avaliação e ações tomadas pelo aprendiz. Quando o aprendiz faz uma ação, isto corresponde a uma transição que leva a um novo estado que pode ser correto ou incorreto, e o aprendiz recebe este feedback (novo estado). Neste caso, DEVS e DFA são utilizados para modelar estados e transições bem definidas (o primeiro quando 
envolve tempo e o segundo quando não), e FIS para modelar transições imprecisas (por exemplo, em um incêndio).

O MI programa de treinamento- avaliação [Rocha, 2014; Rocha, et al. 2011; Rocha e Araujo, 2013] é um modelo de avaliação durante o programa de treinamento que integra diversos tipos de avaliação de desempenho humano e do próprio programa de treinamento (para validar sua efetividade educacional), com as teorias de Kolb [1985] (aprendizagem experiencial e estilos de aprendizagem), e a taxonomia de Bloom [1956] (para estruturação das atividades). Ele possibilita a integração de diferentes atividades e tipos de avaliação [Miller, Imrie e Cox, 1998; Piskurich, Beckschi e Hall, 2000; Salas et al., 2009; Kirkpatrick e Kirkpatrick, 2006] e feedback [Salas et al., 2009; Salas e Rosen, 2007] ao longo do processo de treinamento (fases do jogo), com o uso de diferentes recursos (simulações 3D, jogos simples 2D, vídeos e um formulário de avaliação), conforme Quadro 1.

Quadro 1. Fases do jogo sério criado com base no modelo integrador avaliações - programa de treinamento [adotado de Rocha, 2014].

\begin{tabular}{|c|c|c|c|c|c|}
\hline Critérios & $\begin{array}{c}\text { Descrever } \\
\text { objetivos com } \\
\text { desafios claros e } \\
\text { graduais }\end{array}$ & $\begin{array}{c}\text { Criar atividades que } \\
\text { explorem } \\
\text { aprendizagem } \\
\text { experiencial e } \\
\text { diversificada }\end{array}$ & $\begin{array}{c}\text { Avaliar } \\
\text { desempenho } \\
\text { humano de } \\
\text { modo amplo }\end{array}$ & $\begin{array}{c}\text { Avaliar se } \\
\text { Programa de } \\
\text { Treinamento } \\
\text { satisfaz objetivos }\end{array}$ & $\begin{array}{l}\text { Fornecer } \\
\text { Feedback }\end{array}$ \\
\hline $\begin{array}{l}\text { Fases } \\
\text { /Ref. }\end{array}$ & $\begin{array}{l}\text { Níveis da } \\
\text { taxonomia de } \\
\text { Bloom }\end{array}$ & $\begin{array}{c}\text { Modos de } \\
\text { aprendizagem de Kolb } \\
\text { e aprendizagem } \\
\text { experiencial }\end{array}$ & $\begin{array}{l}\text { Tipos de } \\
\text { avaliação }\end{array}$ & $\begin{array}{l}\text { Níveis de } \\
\text { avaliação de } \\
\text { Kirkpatrick }\end{array}$ & $\begin{array}{l}\text { Dimensões de } \\
\quad \text { feedback }\end{array}$ \\
\hline 1 & 1: lembrar & $\begin{array}{l}\text { experiência concreta } \\
\text { (fazer e sentir) }\end{array}$ & diagnóstica & \multirow{6}{*}{ 2: aprendizado } & $\begin{array}{c}\text { construtivo e } \\
\text { descritivo }\end{array}$ \\
\hline 2 & 1: lembrar & $\begin{array}{l}\text { conceitualização } \\
\text { abstrata (pensar) }\end{array}$ & \multirow{3}{*}{ formativa } & & construtivo, \\
\hline 3 & 2: compreender & \multirow{2}{*}{$\begin{array}{l}\text { experiência ativa } \\
\text { (planejar e fazer) }\end{array}$} & & & prescritivo \\
\hline 4 & 3: aplicar & & & & \\
\hline 5 & 4: analisar & \multirow{2}{*}{$\begin{array}{l}\text { refletir a realidade } \\
\quad \text { (observar) }\end{array}$} & \multirow{2}{*}{ somativa } & & construtivo, e \\
\hline 6 & 5: sintetizar & & & & \\
\hline 7 & 6: avaliar & - & autoavaliação & 1: reação & - \\
\hline
\end{tabular}

A fases 1 e 2 devem explorar o nível 1 da taxonomia de Bloom, descrevendo objetivos que possibilitem lembrar o protocolo treinado. A fase 1 deve ser diagnóstica e possibilitar o aprendiz fazer as ações a partir de conhecimentos prévios e ver/sentir os resultados (experiência concreta); ao passo que a fase 2 deve incluir atividades que permitam a comparação e generalização de fatos e elementos da realidade (conceitualização abstrata) e fornecer feedback prescritivo além do descritivo. As atividades nas fases 3 e 4 devem possibilitar o aprendiz planejar e fazer ações a partir dos conhecimentos refletidos (experiência ativa). Nelas, o aprendiz deve poder compreender e aplicar estes conhecimentos e obter feedback prescritivo (como deveria ter realizado) além do descritivo (acertos e erros). As fases 5 e 6 devem possibilitar uma avaliação somativa, e o aprendiz deve analisar e sintetizar os conhecimentos praticados a partir da observação e reflexão. A última fase compreende a avaliação por parte do aprendiz, ele deve avaliar o seu treinamento e o programa de treinamento em si. 
CBIE-LACLO 2015

Anais dos Workshops do IV Congresso Brasileiro de Informática na Educação (CBIE 2015)

\subsection{Uso da metodologia DevJSTA}

Ao longo do desenvolvimento da metodologia foram criados diferentes protótipos de treinamentos (provas de conceito), usando protocolos operacionais de preparação e resposta a emergência. Inicialmente foram analisadas e especificadas, pela pesquisadora responsável, seis diferentes simulações de treinamento. Depois foram projetadas e modeladas 12 simulações em uma disciplina ofertada em 2012 (10 operacionais que vão desde salvamentos a combates a incêndio e duas simulações de estado das vítimas) e mais oito em 2013 (dois operacionais, dois de comando e três para educação de crianças no trânsito). Os treinamentos foram diversificados para permitir que a metodologia contemplasse diferentes domínios. Por fim, foi desenvolvido um jogo sério completo com sete fases, nomeado JSTA GLPSobControle. Este jogo sério foi utilizado e validado pelos bombeiros (oficiais e praças que tem experiência no domínio) em um estudo de caso, por meio dos dados coletados durante o treinamento pelo próprio jogo sério e por questionários, conforme será descrito na seção 4.2. Dois exemplos de cenários desenvolvidos usando a metodologia criada são descritos a seguir.

O primeiro é uma simulação simples $2 \mathrm{D} / 3 \mathrm{D}$, para celulares com o sistema operacional Android, para controle do painel da viatura do Corpo de Bombeiros (operação da bomba de água), conforme Figura 3 [Lima e Araujo, 2011]. Foi implementado com o motor de jogos jPCT-AE, com programação em Java.

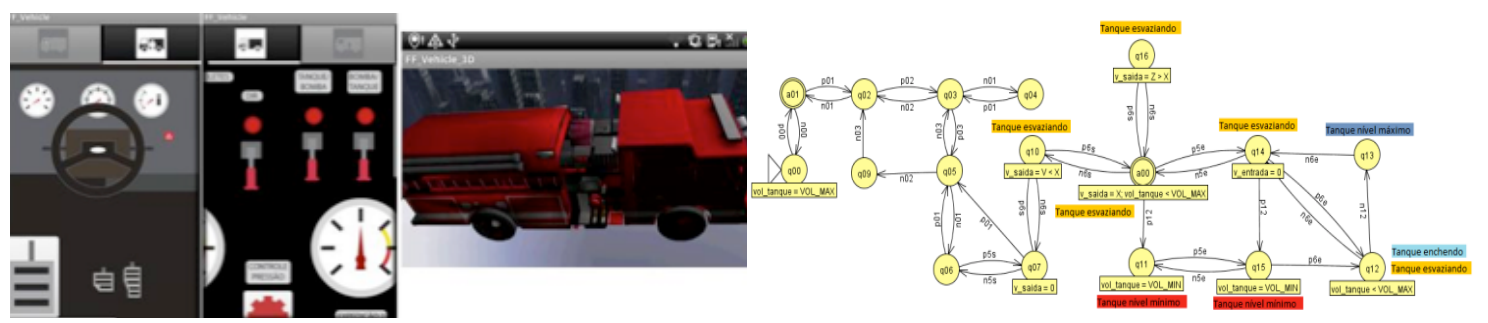

Figura 3. Simulação criada para celulares Android e seu DFA [adotado de Lima e Araujo, 2011].

O segundo exemplo é um jogo sério completo, com três fases $2 \mathrm{D}$ e três fases $3 \mathrm{D}$, multiplataforma, com acesso on-line via navegador $W e b$, para controle de vazamento de gás de cozinha, nomeado GLPSobControle, conforme Figura 4 [Rocha, 2014]. Ele foi implementado com o uso do motor de jogos Unity $3 D \mathbb{C}$, em conjunto a linguagem C\# para programação da simulação. Modelos 3D foram reusados a partir do repositório do 3 WWarehouse $\mathbb{C}$. Foram criadas animações simples para as portas e o quadro de energia, e usados sistemas de partículas do próprio Unity $3 D$ para representar a explosão e o vazamento de gás. Áudios e texturas foram reusadas de repositórios gratuitos encontrados na Internet. O banco de dados foi desenvolvido em linguagem MySQL, com acesso implementado em PHP.

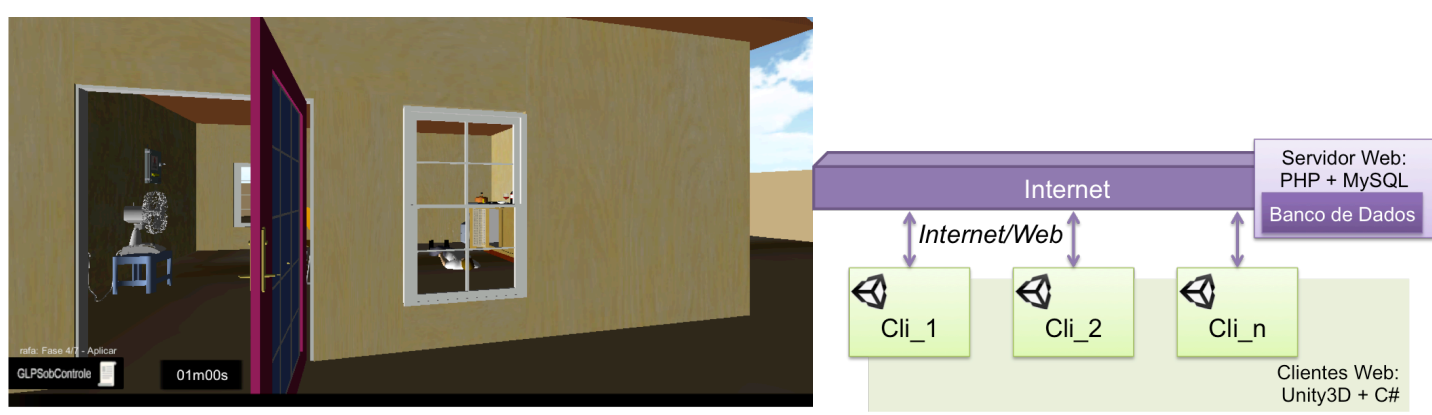

Figura 4. Jogo sério multiplataforma acessado via Web [adotado de Rocha, 2014]. 
CBIE-LACLO 2015

Anais dos Workshops do IV Congresso Brasileiro de Informática na Educação (CBIE 2015)

\section{Avaliações}

Considerando que o trabalho realizado necessita ser avaliado de modo sistêmico, i.e., deve ser considerado todos os elementos envolvidos e sua complexidade e interdependência, e dada a subjetividade do contexto estudado, a abordagem da pesquisa é qualitativa pois visa descrevê-la e não quantificá-la ou classificá-la com métodos estatísticos. Desta forma, foram realizadas avaliações sobre os diversos objetos avaliados (metodologia, arquitetura de suporte, protótipos e jogo sério desenvolvido com ela), para então concluir sobre a viabilidade da metodologia proposta.

\subsection{Avaliação da Metodologia DevJSTA}

A metodologia DevJSTA foi avaliada com especialistas por meio de entrevistas semiestruturadas. A escolha dos entrevistados desta avaliação foi feita por julgamento da pesquisadora (amostra não probabilística de membros da população de potenciais interessados na pesquisa). O julgamento foi feito avaliando as experiências acadêmicas e de atuação nas áreas inter-relacionadas a da pesquisa (todos possuem uma visão holística das áreas integradas) e a disponibilidade e aceitação em participar.

Os entrevistados, no total de cinco pessoas (quatro homens e uma mulher), são três pesquisadores doutores e dois produtores e desenvolvedores de jogos (que possuem especialização ou mestrado). Todos são graduados há mais de 10 anos.

Em relação aos resultados, os pesquisadores entrevistados questionaram mais detalhes sobre alguns conceitos utilizados ou pertinentes ao projeto; deram algumas sugestões para melhoria da metodologia criada ou como trabalhos futuros; e opinaram sobre a relevância da tese e de sua avaliação. Já os desenvolvedores questionaram detalhes sobre a metodologia e o jogo sério desenvolvido, e ressaltaram a relevância do trabalho (inclusive porque passaram por alguns dos problemas apresentados em um projeto de desenvolvimento de um simulador).

A avaliação pelos diversos especialistas serviu para ratificar a importância da metodologia sendo desenvolvida, reforçar os trabalhos futuros, e orientar algumas melhorias ou considerações a serem feitas na sua especificação e no jogo criado. A apresentação do trabalho para esses especialistas também possibilitou a identificação de parcerias futuras para o desenvolvimento de pesquisas e produtos. Além disto, todos os entrevistados responderam que a metodologia orienta o trabalho desempenhado pelos papéis da especialidade do pesquisador no desenvolvimento do JSTA. Dessa forma, há indícios que a metodologia consegue minimizar a distância entre as áreas de conhecimento envolvidas.

\subsection{Avaliação do Jogo Sério GLPSobControle}

O JSTA GLPSobControle foi validado e avaliado pelos bombeiros, no que tange aos aspectos de fidelidade do modelo de simulação e conteúdo, aspectos educacionais de desempenho humano e treinamento das competências intencionadas, bem como a percepção disto pelos aprendizes.

Em um primeiro momento, foram feitas avaliações e validações por dois oficiais bombeiros: do modelo de simulação de controle de vazamento de GLP; do jogo completo GLPSobControle; e do questionário utilizado para avaliação da reação do aprendiz e autoavaliação. As validações foram exitosas, inclusive possibilitaram 


\section{CBIE-LACLO 2015}

Anais dos Workshops do IV Congresso Brasileiro de Informática na Educação (CBIE 2015)

verificar uma oportunidade de melhoria no protocolo que não tinha sido observada anteriormente (somente com o documento que descreve o procedimento). Isto ocorre quando a vítima e o recipiente de gás estão próximos ou o recipiente é alcançado antes da vítima. No protocolo, a sequência de ações é salvar vítimas e depois cortar o fluxo de gás. Além disto, é descrita a prioridade de remover a vítima antes de qualquer outra ação. Entretanto, quando as situações descritas anteriormente acontecem, é mais seguro cortar o fluxo de gás antes de salvar as vítimas. Dessa forma, esta sequência foi alterada no modelo de simulação e será atualizada no protocolo do Corpo de Bombeiros.

Depois disto, os treinamentos foram realizados com uma amostra intencional de oito bombeiros (que abrange os perfis que utilizarão o jogo validado). Quanto a validade do conteúdo pelos aprendizes, ela foi realizada por meio de um questionário e entrevistas abertas informais. Esta validação tem dois objetivos: (1) validar a representação física e comportamental da simulação criada (artefato final criado); e (2) verificar se os aprendizes conseguem relacionar o treinamento à realidade, por meio da autoavaliação. Após a realização do treinamento e coleta de dados (por meio do jogo sério e do questionário), os resultados foram organizados e analisados (conforme descrito em Rocha [2014]).

Segundo o método de transferência inversa descrito por Korteling, Oprins e Kallen [2009], o programa de treinamento é válido e tem efetividade educacional externa se profissionais experientes na prática conseguem utilizar o jogo sério com sucesso. Metade dos profissionais que realizaram o treinamento com sucesso são praças que tem mais de 10 anos de experiência e atenderam mais de cinco vazamentos de GLP. Estes profissionais concordam que conseguem relacionar o que treinaram com a realidade e que o treinamento contribuiu para relembrar e praticar o protocolo. Eles ressaltaram também a necessidade de treinamento constante pois são muitos os Procedimentos Operacionais Padrão e com o tempo os detalhes dos procedimentos acabam esquecidos.

Dessa forma, o pressuposto é que se os profissionais experientes conseguiram utilizar e relembrar o protocolo, então a transferência ocorrerá com os aprendizes que completaram com sucesso este treinamento e com outros que o utilizarem com a supervisão e auxílio do instrutor. Assim, o JSTA GLPSobControle tem indícios de possuir validade educacional externa, porém é necessário a sua aplicação em novos casos e o acompanhamento dos desempenhos dos aprendizes durante um tempo após o treinamento para a comprovação dos efeitos imediatos e em longo prazo para a corporação e assim a confirmação desta validade.

\subsection{Considerações Finais sobre as Avaliações}

Como resultados e indícios da validade representacional e educacional do JSTA, o jogo sério serviu de treinamento das competências propostas e para melhorar o protocolo a partir do modelo de simulação criado. Além disso, foi possível aprimorar o jogo durante os processos de desenvolvimento e validação, além de planejar alterações para uma futura versão e também em trabalhos futuros.

De modo geral, as avaliações do jogo e da metodologia foram positivas, e há indícios da viabilidade da metodologia DevJSTA. Os pesquisadores e desenvolvedores corroboraram com as vantagens de utilizar a metodologia e os oficiais e bombeiros com os benefícios que ela promoveu ao JSTA criado. 


\section{Conclusões e Trabalhos Futuros}

Este trabalho se insere na pesquisa e prática em áreas que apoiam a criação de jogos sérios; de modo a entender como estes jogos devem ser planejados, desenvolvidos, avaliados, validados e utilizados com o propósito de aprendizagem/ treinamento e avaliação do desempenho do aprendiz. A principal contribuição da metodologia e modelos criados é a integração de diferentes áreas para abranger os requisitos relacionados ao treinamento e avaliação do aprendiz por meio do uso do jogo sério. A falta de integração destas áreas, lacuna identificada nos trabalhos relacionados, foi o principal desafio pesquisado. A segunda contribuição é que a metodologia possibilita a análise do protocolo operacional padrão por meio da simulação, e inclusive foi possível identificar uma oportunidade de melhoria no protocolo usado no jogo sério criado. A terceira contribuição é a especificação de cada processo (atores e papéis, atividades e artefatos) e sua exemplificação de uso com o desenvolvimento do JSTA completo GLPSobControle, geralmente os trabalhos relacionados não detalham seus processos.

Como trabalho futuro, a metodologia será avaliada quali e quantitativamente por outros desenvolvedores, e os jogos sérios desenvolvidos deverão ser utilizados por um número maior de aprendizes, por um período de tempo mais longo. Outros trabalhos podem ser realizados para melhorar ou estender as soluções propostas, como por exemplo, integrar e melhorar as soluções de interoperabilidade e simulação distribuída em jogos sérios, ou estender a avaliação para compreender também as habilidades não técnicas, ou ainda criar ferramentas para auxiliar a metodologia e/ou arquitetura. Além disto, podem ser realizados estudos comparativos com as soluções propostas, como por exemplo, criar um novo programa de treinamento fundamentado em outras teorias pedagógicas e compará-lo com o programa desenvolvido neste trabalho.

\section{Agradecimentos}

Este projeto foi financiado pelo CNPq (Processo 480291/2012-8), FAPESP e CAPES (Projeto INCT-SEC, processos 2010/07179-8, 08/57870-9, 237216/2012-4, 143567/2011-0, 573963/2008-8). Os autores gostariam de agradecer ao Corpo de Bombeiros por seu apoio, bem como aos alunos de Iniciação Científica e graduação.

\section{Referências}

ABNT. (2001). NBR ISO 10015:2001- Gestão da Qualidade: diretrizes para treinamento. RJ: ABNT.

ADAMS, W.K.; et al. (2007). A Study of Educational Simulations Part I - Engagement and Learning. Journal of Interactive Learning Research, v. 12. p. 397-419.

AKILLI, G.K.; CAGILTAY, K. (2006). An Instructional Design/Development Model for the Creation of Game-Like Learning Environments: the FIDGE model. In: Pivec, M. (Ed.), Affective and Emotional Aspects of Human-Computer Interaction: Game-Based and Innovative Learning. IOS Press, p. 93-112.

ASLAN, S.; BALCI, O. (2015). GAMED: Digital Educational Game Development Methodology, In: Simulation, v. 91, n. 4, p. 307-319.

BALCI, O. (2012). A Life Cycle for Modeling and Simulation. Simulation, v. 88, n. 7. p. 870-883.

BANKS, J.; et al. (2001). Discrete-Event System Simulation. 3rd. ed. New Jersey: Prentice-Hall.

BECKER, K.; PARKER, J. (2012). Serious Instructional Design: ID for digital simulations and games. In: P. RESTA (ED.), Proceedings of Society for Information Technology \& Teacher Education International Conference 2012. Chesapeake, VA: AACE. p. 2480-2485.

BLOOM, B.S. (1956). Taxonomy of Educational Objectives: the classification of educational goals - Handbook I: Cognitive Domain. New York: McKay. 
CHANDLER, H.M. (2012). Manual de Produção de Jogos Digitais. 2. ed. Porto Alegre: Bookman. DICK, W.; CAREY, L. CAREY, J.O. (2004). The Systematic Design of Instruction. 6th ed. Allyn \& Bacon.

FORD, F. (2004). The Euclid RTP 11.13 SE Development \& Exploitation Process (SEDEP). European Simulation Interoperability Workshops, 04E-SIW- 037, Edinburgh, Scotland. 10p.

FREITAS, S.; JARVIS, S. (2006). A Framework for Developing Serious Games to meet Learner Need. Interservice/Industry Training, Simulation, and Education Conference. 11p.

GODOY, A.; BARBOSA, E.F. (2010). Game-scrum: an approach to agile game development. In: Simpósio Brasileiro de Jogos e Entretenimento Digital. p. 292-295.

HAYS, R.T. (2005). The Effectiveness of Instructional Games: a literature review and discussion. Naval Air Warfare Center Training Systems Division (Technical Report 2005 - 004).

IEEE. (2003). 1516.3-2003- IEEE Recommended Practice for High Level Architecture (HLA): federation development and execution process (FEDEP). New York: IEEE.

IEEE. (2010). 1730-2010- Distributed Simulation Engineering and Execution Process (DSEEP). Washington: IEEE.

KIRKPATRICK, D.L.; KIRKPATRICK, J.D. (2006). Evaluating Training Programs: The Four Levels. 3rd Edition. San Francisco, CA, USA: Berrett-Koehle. 568 p.

KIRKLEY, S.; TOMBLIN, S.; KIRKLEY, J. (2005). Instructional Design Authoring Support for the Development of Serious Games and Mixed Reality Training. I/ITSEC. Bloomington.

KOLB, D.A. (1984). Experiential Learning: Experience as The Source of Learning and Development. Englewood Cliffs: Prentice-Hall Inc. p. 20-38.

KORTELING, J.E., OPRINS, E.A.P.B.; KALLEN, V.L. (2012). Measurement of Effectiveness for Training Simulations. In: RTO System Analysis and Studies Panel (SAS), RTO-MP-SAS-095. Amsterdam, The Netherlands. p. 1-14.

LAUBISCH, A.; CLUA, E. (2010). Scrum4Games: Uma aplicação do Scrum para projetos de games focada em game design. In: IX Proceedings do SBGames 2010, Florianópolis, p. 178-187.

LIMA, R. H. P.; ARAUJO, R. B. (2011). Interface de Visualização 3D em Dispositivos Móveis para Simulações de Treinamento. Relatório Científico (Bolsa FAPESP de Iniciação Científica) Departamento de Computação, Universidade Federal de São Carlos, São Carlos, SP.

MILLER, A.; IMRIE, B.W.; COX, K. (1998). Functions of Assessment. In:____. Student Assessment in Higher Education: a handbook for assessing performance. London: Kogan Page. p. 23-40.

NOVAK, J. (2010). Desenvolvimento de Games. São Paulo: Cengage. 443 p.

PISKURICH, G.; BECKSCHI P.; HALL, B. (2000). The ASTD Handbook of Training Design and Delivery: a comprehensive guide to creating and delivering training programs: instructor-led, computer-based, or self-directed. 2nd ed., New York: McGraw-Hill.

ROCHA, R.V. (2014). Metodologia iterativa e modelos integradores para desenvolvimento de jogos sérios de treinamento e avaliação de desempenho humano. Tese (Doutorado em Ciência da Computação) - Departamento de Computação, Universidade de São Carlos, São Carlos, SP.

ROCHA, R.V.; ARAUJO, R.B. (2013). Avaliação de Desempenho Humano Como Parte Integrada da Metodologia de Criação de Jogos Sérios para Treinamento. In: $24^{\circ}$ Simpósio Brasileiro de Informática na Educação (SBIE 2013), Campinas. p. 144-153.

ROCHA, R.V.; et al. (2011). Sistema Integrado para Avaliação de Desempenho Humano em Simulações Interativas. In: 22 Simpósio Brasileiro de Informática na Educação (SBIE 2011), Aracaju.p. 436-445.

RODRIGUES, H.F.; MACHADO, L.S., VALENÇA, A.M. (2010). Definição e Aplicação de um Modelo de Processo para o Desenvolvimento de Serious Games na Área de Saúde. In: Proc. Congresso da Sociedade Brasileira de Computação - Workshop de Informática Médica. p. 15321541 .

SCHUYTEMA, P. (2008). Design de Games: uma abordagem prática. SP: Cengage Learning. 447 p. VAN DER ZEE, D.J.; HOLKENBORGB, B.; ROBINSON, S. (2012). Conceptual modeling for simulation-based serious gaming. In: Decision Support Systems, v. 54. p. 33-45.

ZIN, N.A.M; JAAFAR, A.; YUE, W.S. (2009). Digital Game-Based Learning (DGBL) Model And Development Methodology For Teaching History. WSEAS Transactions On Computers, v.2, n.8. p. 322-333. 